\title{
A giant bulla case that developed in the late period after Covid 19: a case report
}

\author{
Bayram Metin ${ }^{1}$, Mehmethan Turan², Emel Eskitaşcıoğlu ${ }^{3}$ \\ ${ }^{1}$ Thoracic Surgery Department, Kayseri Acibadem Hospital, Kayseri, Turkey \\ ${ }^{2}$ Chest Diseases Department, Private Acibadem Kayseri Hospital, Kayseri, Turkey \\ ${ }^{3}$ Department of Anesthesiology and Reanimation, Private Acibadem Kayseri Hospital, Turkey
}

Received: 2021-03-06.

Accepted: 2021-05-07

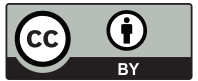

This work is licensed under a Creative Commons Attribution 4.0 International License
J Clin Med Kaz 2021; 18(3):68-70

\section{Corresponding author:}

Bayram Metin.

E-mail: drbaymet@hotmail.com

\begin{abstract}
Covid 19 patients' lung imaging, parenchymal pneumonic opacity areas with ground glass density, mostly located in the subpleural regions, are observed. With the increase in the number of cases, different lung complications have begun to be seen in cases other than pneumonic consolidations. Here, we wanted to emphasize the necessity of followup of Covid 19 patients after discharge by presenting our case who developed giant bulla in the late period, but not in the early follow-up after Covid 19.
\end{abstract}

Key words: giant Bulla, Covid 19, thoracoscopy

\section{Introduction}

The new type of Coronavirus 19 (Covid-19) disease was first seen in Wuhan, China, in December 2019. By showing human-to-human transmission, it quickly took hold of the whole world. In these patients' lung imaging, parenchymal pneumonic opacity areas with ground glass density, mostly located in the subpleural regions, are observed. [1]. With the increase in the number of cases, different lung complications have begun to be seen in cases other than pneumonic consolidations. Here, we wanted to emphasize the necessity of follow-up of Covid 19 patients after discharge by presenting our case who developed giant bulla in the late period, but not in the early follow-up after Covid 19.

\section{Case presentation}

Our 51-year-old male patient was admitted to the hospital on 01.09.2020 with complaints of cough, shortness of breath, joint pain, fever, and Oronasal Covid-19 Polymerase chain reaction (PCR) test came out positive. On physical examination, breathing sounds could not be detected in the left basales. Oxygen saturation at room air was $90 \%$. He has never smoked. There was no known history of asthma or COPD.

The patient's blood test results are as follows: leukocyte $5.79 \times 10^{3} / \mathrm{uL}$, neutrophils $83.5 \%$, lymphocytes: $13 \%$, hemoglobin $14.6 \mathrm{~g} / \mathrm{dl}$, platelets: $16500010^{3} / \mathrm{uL}$
LDH: 730 IU/L CRP: 146.9 mg/dl, Ferritin 1420 mg/l. In Thoracic Tomography Peripherally located infiltrations with ground glass density were observed in the upper, middle, and lower lobes of both lungs and the appearance was primarily evaluated in favor of viral pneumonia due to Covid 19 (Figure 1).

Figure 1 - Covid 19 pneumonic infiltrations

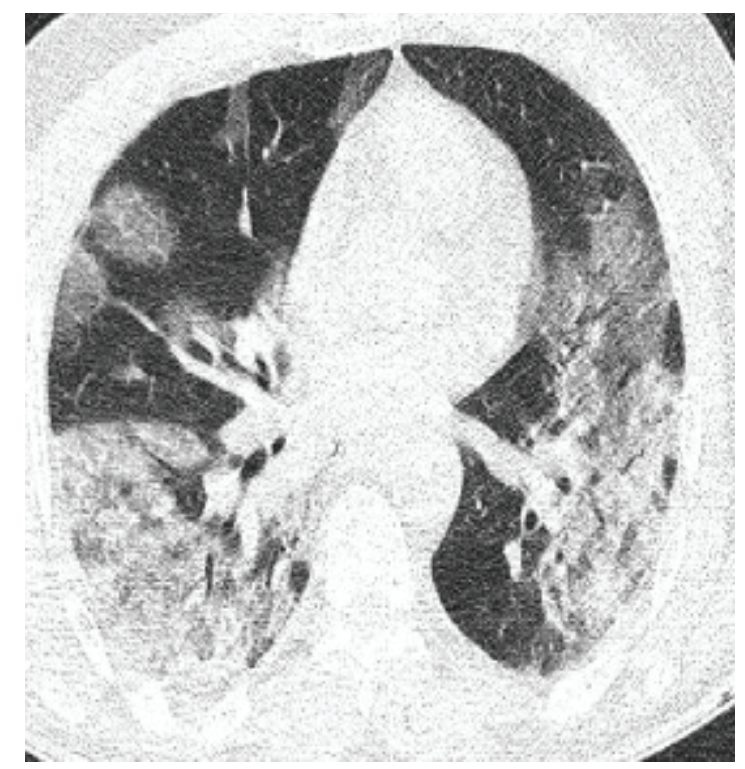

Journal of Clinical Medicine of Kazakhstan: 2021 Volume 18, Issue 3 
Figure 2 - Chest radiography of After ten day than Covid 19

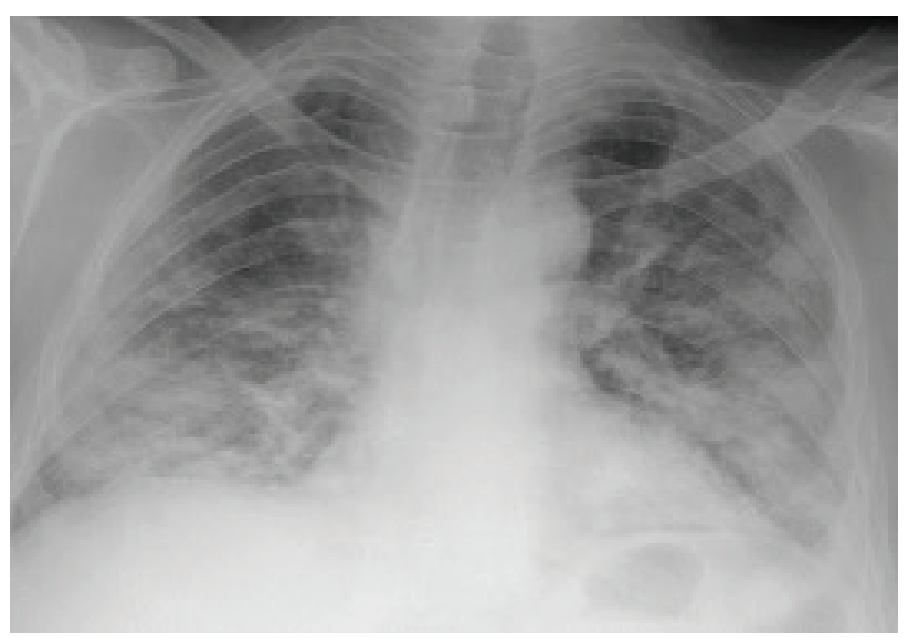

Figure 3 - Thorax bt and paac graphs taken in the 5th month

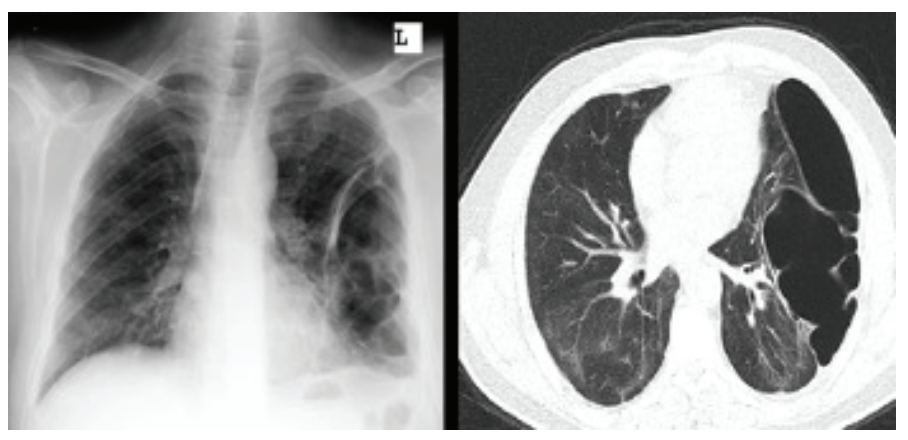

Figure 4 - Operation images

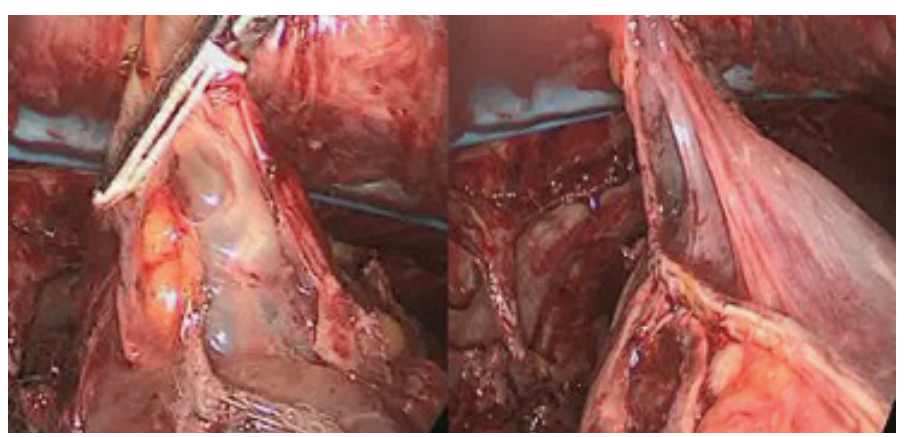

He was hospitalized for about one week and received medical support in nasal oxygen support, Plaquenil, Favipavir, Methylprednisolone, Enoxaparin, and antibiotherapy. When the result of the Covid PCR test, which was taken twice during the patient's follow-up, was negative, and when the blood values improved, he was discharged and completed the quarantine period at home. A partial decrease in ground glass densities was detected in the control chest X-ray ten days after discharged, and his follow-up was continued (Figure 2).

At the 5th month of the patient, bullous formations with a diameter of about $16 \times 8 \times 17 \mathrm{~cm}$ continuing along the upper lobe of the left lung, lingula, and lower lobe superior segments were noted in the Thorax CT performed due to chest pain (Figure 3).

Thereupon, surgery was recommended to the patient, stating a risk of bullae growth and explosion. With the uniportal video thoracoscopic approach, the giant bullous structure originating from the lower lobe superior segment of the left lung was removed by wedge resection (Figure 4).

In the pathological examination of the extracted specimen, typical features for Covid-19 histopathology, vascular thrombi, and vasculitis findings were not discovered. However, extensive perivascular lymphocytic infiltrations were observed in small diameter vessels.

The patient, who stayed in the hospital for two days after surgery, was discharged without any problems after the drain was removed.

\section{Discussion}

As the new type of coronavirus became widespread worldwide, cases such as pneumatocele, giant bulla development, mediastinal emphysema, and pneumothorax development have started to be reported in some published cases [1]. Although the exact mechanism of bulla formation is not fully understood, it is thought that it may be due to the patients' underlying emphysema, previous air space, or cystic changes in the parenchyma [2]. Besides, since high flow oxygen therapy, positive pressure mechanical ventilation with CBAP or intubation is applied to patients desaturated during the Covid process, there is a possibility of alveolar damage and bulla formation pneumothorax. It has been reported that Covid 19 pneumonia itself causes cystic changes in the parenchyma by causing alveolar damage [3-4]. Bilateral diffuse alveolar damage desquamates in pneumocyte cells, and hyaline membrane formation has been reported in autopsy studies in patients with Covid 19 who died. Thrombosis, microangiopathy, leukocyte infiltration consisting mostly of lymphocytes have been frequently reported. It has been stated that diffuse alveolar damage may develop due to endothelial and vascular damage caused by microthrombi [3]. It is predicted that air cysts resulting from alveolar damage may coalesce to form pneumotoceles, and these pneumotoceles may rupture, merge and grow, and thus giant bullae may form. As a result of the explosion of these giant bullae, complications such as pneumothorax and pneumomediastinum have been reported to be possible to develop [2-4]. In the pathological specimen examination of our patient, vascular thrombi and vasculitis findings were not discovered. However, extensive perivascular lymphocytic infiltrations were observed in small diameter vessels.

In one of the published cases, they stated that CBAP and high-flow oxygen were applied to the patient during the Covid-19 process and that they could not fully evaluate whether the pneumothorax developed in this process was related to this [5]. Yaskawa et al. stated that in their patient hospitalized for Covid pneumonia for ten days with nasal oxygen therapy, tension pneumothorax developed in the right hemithorax on the 14th day after discharge. They stated that they observed bilateral infiltrations and giant bulla formation at the right middle lobe level in the thorax CT imaging performed after the drain was inserted [4].

While there was no bulla formation in the initial period of Covid disease in a case published by Berhane et al., it was stated that giant bullae developed in the right hemithorax during the follow-up of the patient who received CBAP treatment for eight days due to severe pneumonia and desaturation due to Covid 19 [5].

In most of the published cases, it is seen that the development of complications in the form of bulla and pneumothorax is encountered during the hospitalization period due to Covid 19 or during the early recovery period [1-5]. However, in publications, it has begun to be stated that Covid 19 patients with severe lung involvement and positive pressure respiratory support with mechanical ventilation or non-invasive mechanical ventilation should be followed up with tomography to develop bulla pneumothorax after treatment [6]. 
In this article, we wanted to present our case whose giant bulla was detected in the 5 th month after the recovery of Covid-19 disease. While the development of giant bulla or pneumothorax in the majority of the cases that have been included in the literature so far occurred either during the active disease period in which Covid 19 patients were hospitalized or during the early discharge after recovery, a case with a giant bulla in the 5th month, like ours, has not been reported in the literature yet. Based on this case, we would like to emphasize that cases with severe pneumonic involvement during the Covid 19 disease period should be evaluated with a control tomography image at least six months after discharge.

Disclosures: There is no conflict of interest for all authors.
Acknowledgements: None.

Funding: None.

\section{References}

1. Sun R, Liu H, Wang X. Mediastinal Emphysema, Giant Bulla, and Pneumothorax Developed during the Course of COVID-19 Pneumonia. Korean J Radiol. 2020; 21(5):541-544. https://doi.org/10.3348/kjr.2020.0180

2. O.M.P. Jolobe, Air leaks, pneumatoceles, and air spaces in Covid-19 pneumonia. American Journal of Emergency Medicine. https:// doi.org/10.1016/j.ajem.2020.08.098

3. Janssen ML, Van Manen MJG, Cretier SE, Braunstahl G-J. Pneumothorax in patients with prior or current COVID-19 pneumonia. Respiratory Medicine Case Reports. 2020; 31. https://doi.org/10.1016/j.rmcr.2020.101187

4. KYasukawa K, Vamadevan A, and Rollins R. Bulla Formation and Tension Pneumothorax in a Patient with COVID-19. Am. J. Trop. Med. Hyg. 2020; 103(3):943-944 https://doi.org/10.4269/ajtmh.20-0736

5. Berhane S, Tabor A, Sahu A, Singh A.Development of bullous lung disease in a patient with severe COVID-19 pneumonitis. BMJ Case Rep. 2020; 13:e237455. doi:10.1136/bcr-2020-237455

6. Xu W, Luo X, Wang H, Shen C, Song Y, Sun T, Chen M, Pulmonary emphysema, bullae, and pneumothorax in COVID-19 pneumonia. Radiology Case Reports. 2021; 16:995 - 998 